\title{
FGFR1 is amplified during the progression of in situ to invasive breast carcinoma
}

\author{
Min Hye Jang ${ }^{1,2}$, Eun Joo Kim³ ${ }^{3}$ Yoomi Choi ${ }^{1,2}$, Hee Eun Lee ${ }^{2}$, Yu Jung Kim ${ }^{4}$, Jee Hyun Kim, Eunyoung Kang ${ }^{4}$, \\ Sung-Won $\mathrm{Kim}^{4}$, In Ah $\mathrm{Kim}^{4}$ and So Yeon Park ${ }^{1,3,4^{*}}$
}

\begin{abstract}
Introduction: Gene amplification is an important mechanism for activating oncogenes in malignant tumors. Although amplification of HER2, C-MYC, CCND1 and FGFR1 has been reported in breast cancers, their role in the progression of in situ to invasive breast carcinoma is unclear. To investigate this question we compared the amplification frequencies of these genes in pure ductal carcinoma in situ (DCIS), DCIS associated with invasive carcinoma, and invasive carcinoma.
\end{abstract}

Methods: We performed fluorescence in situ hybridization of the selected genes on tissue microarrays composed of 179 pure DCIS and 438 invasive carcinomas. Two hundred and sixteen of the latter had DCIS components, and in those cases we compared gene amplification in the intraductal and invasive components of each carcinoma.

Results: The rate of amplification of FGFR1 was higher in invasive carcinomas than in the pure DCIS, but the opposite was true for HER2 amplification. These findings applied consistently to high-grade tumors, but not to low/ intermediate-grade tumors. The amplification status of HER2, C-MYC, CCND1 and FGFR1 was generally similar in the matched invasive and DCIS components of the same tumors. However, FGFR1 amplification was more common in the invasive components than in the DCIS components. In survival analyses, FGFR1 amplification was found to be an independent prognostic factor for poor disease-free survival for all patients with invasive carcinoma and for the hormone receptor-positive subgroup.

Conclusion: Amplification of HER2, C-MYC and CCND1 seems to play a role in the early development of breast cancer, but not in its progression. However, the increased frequency of FGFR1 amplification in invasive carcinomas compared with pure DCIS and in the invasive components of individual tumors, and its association with decreased disease-free survival, suggests a role for FGFR1 amplification in the progression of breast cancer including in situ-toinvasive transition, as well as initiation.

\section{Introduction}

Development of breast cancer depends on the accumulation of a variety of genetic alterations, including activation or amplification of oncogenes [1]. In breast cancer, the most prominent and frequent amplicons have been located at chromosomal positions 1q, 8p12, 8q24, $11 \mathrm{q} 13,12 \mathrm{p} 13,16 \mathrm{p} 13,7 \mathrm{q} 12-21$ and 20q13, and several target oncogenes have been identified [2-5]. The best characterized oncogene is HER2, located at 17q12-21, which is amplified in 15 to $20 \%$ of breast cancers [6,7]. Other oncogenes that are frequently amplified in breast

\footnotetext{
* Correspondence: sypmd@snu.ac.kr

'Department of Pathology, Seoul National University College of Medicine,

103 Daehakro, Jongno-gu, Seoul 110-799, Korea

Full list of author information is available at the end of the article
}

cancer include $C-M Y C$, FGFR1 and CCND1. C-MYC, located at $8 \mathrm{q} 24$, encodes a transcription factor with a basic region/helix-loop-helix/leucine zipper domain, which is a key regulator of cell growth, proliferation, metabolism, differentiation, and apoptosis [8]. The frequency of $C-M Y C$ amplification in breast cancer varies, with an average frequency of $16 \%$ [9]. Although $C-M Y C$ amplification is associated with a risk of relapse and death [9-12], its prognostic role is still unclear. FGFR1 at $8 \mathrm{p} 12$ encodes a tyrosine kinase receptor belonging to the fibroblast growth factor and growth factor receptor family, which is amplified in 9 to $15 \%$ of breast cancers [13-16]. FGFR1 amplification is associated with a poor prognosis in breast cancer $[14,16,17]$. CCND1, located at $11 \mathrm{q} 13$, is an estrogen-responsive gene with oncogenic
C Biomed Central 
potential as it influences the $\mathrm{G}_{1} / \mathrm{S}$ phase transition [18]. CCND1 is amplified in 13 to $20 \%$ of breast cancers $[2,16,19]$.

Ductal carcinoma in situ (DCIS) is an early pathologic stage of breast cancer characterized by proliferation of tumor cells within the ductal-lobular system but not extending through the basement membrane. Like invasive breast cancer, DCIS comprises a highly heterogeneous group of diseases with diverse histologic features, molecular alterations and risks of progression to invasive cancer [20-22]. There appear to be at least two broad groups of DCIS lesions: low-grade DCIS characterized by $16 \mathrm{q}$ loss and 1q gain, and high-grade DCIS displaying complex genetic changes including gains at chromosome locations $8 \mathrm{q}$ and 17q [23-25]. Molecular studies have revealed that in situ lesions preferentially cluster with invasive lesions of the same grade in gene expression profiling, and that the in situ and invasive components of the same tumor exhibit similar patterns of genetic alterations, suggesting that DCIS is a precursor for invasive cancer of similar grade $[23,25,26]$.

The natural history of DCIS is poorly understood, although it is known that 14 to $53 \%$ of in situ lesions evolve to invasive cancer over a period of 10 years or more if left untreated [20]. The mechanisms by which DCIS progress to invasive carcinomas are not well understood, and robust biomarkers capable of stratifying the aggressive forms of DCIS from the indolent forms are lacking. Moreover, the role of gene amplification in the progression of DCIS to invasive breast cancer is uncertain. Some workers found no difference in gene amplification frequencies between DCIS and invasive carcinomas [27-29]. Others have suggested that $C-M Y C$ amplification plays an important role in the transition, because they found amplification only in the invasive component $[30,31]$. However, this finding was not confirmed in other studies [27-29]. Furthermore, there have been few studies comparing amplification of FGFR1 in pure DCIS, DCIS associated with invasive cancer, and invasive breast cancer, although it has been found to be associated with breast cancer progression [14,16,17].

In this study, we compared the gene amplification frequencies of HER2, C-MYC, CCND1 and FGFR1 in a relatively large series of pure DCIS, DCIS associated with invasive carcinoma, and invasive carcinomas, to investigate the role of gene amplification in the progression of DCIS to invasive carcinomas. We also analyzed the gene amplification status of the in situ and invasive components in the invasive carcinomas that were accompanied by DCIS.

\section{Materials and methods}

Tissue specimens

Six hundred and seventeen consecutively resected primary breast cancers including 438 invasive carcinomas and 179 DCIS were collected in Seoul National University Bundang Hospital from 2003 to 2009. Of the 438 invasive breast cancers, 216 cases had enough DCIS component for evaluation. The baseline characteristics of the cases are summarized in Table 1. Clinicopathologic information was obtained by reviewing medical records and $\mathrm{H}$ \& E-stained sections. The following histopathologic variables of the invasive carcinomas were determined: histologic subtype, $\mathrm{T}$ stage, $\mathrm{N}$ stage, Bloom-Richardson histologic grade, lymphovascular invasion, tumor border, and presence or absence of a DCIS component. For DCIS cases, we recorded the extent of tumor, nuclear grade, presence of necrosis and architectural pattern. All cases were independently reviewed by two breast pathologists (SYP and HEL). The study was approved by the institutional review board of Seoul National University Bundang Hospital (Protocol \# B-0909/083-002), waiving the requirement for informed consent for the study.

Table 1 Baseline characteristics of specimens

\begin{tabular}{ll}
\hline Characteristic & $\boldsymbol{n}(\%)$ \\
\hline Pure DCIS & 179 \\
Age (years) & \\
$\quad$ Mean (range) & $50(26$ to 82) \\
Grade & $16(8.9)$ \\
$\quad$ Low & $85(47.5)$ \\
$\quad$ Intermediate & $78(43.6)$ \\
$\quad$ High & 438 \\
Invasive carcinomas & \\
Age (years) & $50(21$ to 87$)$ \\
$\quad$ Mean (range) & \\
Stage & $143(32.6)$ \\
I & $235(53.7)$ \\
II & $55(12.6)$ \\
III & $5(1.1)$ \\
IV & \\
Histologic subtype & $397(90.6)$ \\
Invasive ductal carcinoma & $14(3.2)$ \\
Invasive lobular carcinoma & $12(2.7)$ \\
Mucinous carcinoma & $5(1.1)$ \\
Metaplastic carcinoma & $3(0.7)$ \\
Tubular carcinoma & $7(1.6)$ \\
Others & \\
Histologic grade & $108(20.1)$ \\
Grade I & $148(33.8)$ \\
Grade II & $188(42.9)$ \\
Norade III & $100(4.2)$ \\
Grade of associated DCIS & \\
Intermediate & $(49.1)$ \\
\hline
\end{tabular}

${ }^{\mathrm{a}}$ From 216 ductal carcinoma in situ (DCIS) associated with invasive carcinoma. 


\section{Tissue microarray construction}

We used tissue microarrays (TMAs) to assess the gene amplification status in the collected cases. To overcome sampling errors caused by TMA evaluation, all slides including slides immunohistochemically stained for standard biomarkers were reviewed and the most representative tumor section was selected for each case. If the tumor showed regional differences in histology or biomarker expression, different tumor areas were selected. Three tissue columns of invasive carcinoma and pure DCIS $(2.0 \mathrm{~mm}$ in diameter for invasive carcinomas, $4.0 \mathrm{~mm}$ in diameter for DCIS) were taken from different areas of the tumors and arranged in new TMA blocks using a trephine apparatus (Superbiochips Laboratories, Seoul, Korea). Lots of DCIS associated with invasive carcinoma were not large enough for multi-core construction, and one tissue column of DCIS associated with invasive carcinoma $(4.0 \mathrm{~mm}$ in diameter) was selected. However, because the analysis of DCIS associated with invasive carcinoma in a single TMA core may not be representative, we re-evaluated the discrepant cases for gene amplification between DCIS and invasive components of the same tumor using all tumor sections with a DCIS component.

\section{Fluorescence in situ hybridization assays}

To characterize $C-M Y C$ and $C C N D 1$ amplification, we performed fluorescence in situ hybridization (FISH) analyses on TMA samples with commercially available locusspecific probes and chromosome enumeration probes (CEPs): LSI C-MYC SpectrumOrange probe (8q24.12q24.13) and CEP 8 SpectrumGreen probe (8p11.1-q11.1); and LSI CCND1 SpectrumOrange probe (11q13) and CEP 11 SpectrumGreen probe (11p11.11-q11)] (Abbott Molecular, Downers Grove, IL, USA). HER2 FISH was performed using the PathVysion assay (Abbott Molecular), and FGFR1 amplification was analyzed with locus-specific BAC, RP11-100B16 (chr8:38,358,839-38,522,417) and CEP 8 SpectrumGreen probes (8p11.1-q11.1) (Abbott Molecular). The BAC clone was obtained from Invitrogen (Carlsbad, CA, USA) and purified with a large construction kit (Qiagen, Valencia, CA, USA). DNA from the BAC clone was labeled with SpectrumOrange using a nick translation kit (Abbott Molecular), and the specificity of the BAC probe was verified by metaphase FISH analyses to confirm their proper chromosomal localization and rule out the presence of any cross-hybridization.

FISH was performed as reported for analysis of HER2 amplification [32]. Briefly, $4 \mu \mathrm{m}$ deparaffinized TMA sections were incubated in pretreatment solution (Abbott Molecular) at $80^{\circ} \mathrm{C}$ for 30 minutes, then in protease solution (Abbott Molecular) for 20 minutes at $37^{\circ} \mathrm{C}$. Probes were diluted in tDen-Hyb-2 hybridization buffer (InSitus Biotechnologies, Albuquerque, NM, USA). Co-denaturation of the probes and DNA of the tissue sections was achieved by incubating for 5 minutes $\left(73^{\circ} \mathrm{C}\right.$ for $H E R 2,80^{\circ} \mathrm{C}$ for $C-M Y C$ and $C C N D 1,90^{\circ} \mathrm{C}$ for FGFR1) using HYBrite ${ }^{\mathrm{TM}}$ (Abbott Molecular) followed by 16-hour hybridization at $37^{\circ} \mathrm{C}$. Post-hybridization washes were performed according to the protocols supplied. Slides were mounted in 4',6-diamidino-2-phenylindole/anti-fade and viewed with a fluorescence microscope. Gene signals per cell were evaluated in 50 tumor nuclei for each TMA core. The average gene copy number and gene:CEP ratio was calculated separately for each core, and the tumor was considered amplified if the average gene copy number was $>6.0$ or if the gene: CEP signal ratio was $>2.2$ in at least a core. In addition to the equivocal cases, $10 \%$ of all cases were scored independently by two observers (MHJ and EJK).

\section{Immunohistochemical analyses and scoring}

Expression of standard biomarkers including estrogen receptor (ER), progesterone receptor (PR), HER2, p53 and $\mathrm{Ki}-67$ was evaluated in full sections at the time of diagnosis or in TMA sections for missing data during the study. Epidermal growth factor receptor (EGFR) and cytokeratin 5/6 were evaluated using TMAs. Tissue sections $4 \mu \mathrm{m}$ thick were cut, dried, deparaffinized, and rehydrated following standard procedures. All of the sections were subjected to heat-induced antigen retrieval. Immunohistochemical staining was carried out in a BenchMark XT autostainer (Ventana Medical Systems, Tucson, AZ, USA) using an iView detection kit (Ventana Medical Systems) for ER (1:100, clone SP1; Labvision, Fremont, CA, USA), PR (1:70, PgR 636; Dako, Capinteria, CA, USA), HER2 (1:700, polyclonal; Dako), p53 (1:600, D07; Dako), Ki-67 (1:250, MIB-1; Dako), cytokeratin 5/6 (1:50, clone D5/16 B4; Dako) and EGFR (EGFR pharmDx ${ }^{\text {TM; Dako). }}$

$E R$ and $P R$ were regarded positive if there were at least $1 \%$ positive tumor nuclei, according to the American Society of Clinical Oncology/College of American Pathologists guidelines [33]. Expression of HER2 and EGFR was scored as follows: 0 , no staining; $1+$, weak and incomplete membranous staining in $\geq 10 \%$ of the tumor cells; $2+$, weak to moderate, complete membranous staining in $\geq$ $10 \%$ of the tumor cells; and $3+$, strong, complete membranous staining in $\geq 30 \%$ of the tumor cells. Any positive staining was regarded as positive for EGFR, and 3+ was considered positive for HER2. For cytokeratin 5/6, cases with any positive membranous staining were grouped as positive. For p53, cases with $10 \%$ or more positive staining were grouped as positive. For the Ki-67 proliferation index, cases with $20 \%$ or more positive tumor cells were regarded as having high indices.

\section{Definition of breast tumor subtypes}

Breast cancer subtypes were defined according to Voduc and colleagues [34] with minor modifications, and were categorized as follows: luminal A (ER-positive 
or PR-positive, HER2-negative, Ki-67 < 14\%), luminal B (ER-positive or PR-positive, HER2-negative, Ki-67 $\geq$ 14\%; or ER-positive or PR-positive, HER2-positive), HER2-positive (ER-negative, PR-negative, HER2-positive), basal-like (ER-negative, PR-negative, HER2-negative, cytokeratin 5/6-positive and/or EGFR-positive) and triple-negative, nonbasal (negative for all markers). HER2 positivity was determined from the FISH results.

\section{Statistical analysis}

After omitting all cases in which FISH analysis failed for all the genes (11 cases of invasive carcinoma, 13 cases of DCIS associated with invasive carcinoma and four cases of pure DCIS), a total of 427 invasive carcinomas, 203 DCIS associated with invasive carcinomas and 175 pure DCIS were informative for at least one gene. FISH failures were due to loss of tissue on the TMA, lack of tumor cells in the arrayed tissue or inadequate hybridization. Statistical significance was analyzed using Statistical Package SPSS version 15.0 for Windows (SPSS Inc., Chicago, IL, USA).

The chi-square test or Fisher's exact test was used when comparing gene amplification frequencies between groups and analyzing associations of gene amplification with clinicopathologic characteristics of tumors. The Spearman correlation test was used to analyze the association of co-amplification. For the 203 invasive carcinomas with DCIS components, McNemar tests were used to see whether the (paired) differences between gene amplification in the invasive and DCIS components of the same tumors were significantly different. Survival curves were estimated using the Kaplan-Meier productlimit method, and the significance of differences between survival curves was determined using the log-rank test. Covariates that were statistically significant in the univariate analysis were then included in the multivariate analysis using a Cox proportional hazards regression model; the hazard ratio (HR) and its $95 \%$ confidence interval $(\mathrm{CI})$ were assessed for each factor. $P<0.05$ was considered statistically significant. All $P$ values reported were two-sided.

\section{Results}

Gene amplification in pure DCIS, DCIS associated with invasive carcinomas, and invasive carcinomas

We measured the HER2, C-MYC, CCND1 and FGFR1 amplification status in pure DCIS, DCIS associated with invasive carcinomas, and invasive carcinomas (Figure 1). The frequency of FGFR1 amplification was higher in invasive carcinomas than in pure DCIS $(12.5 \%$ vs. $6.0 \%, P$ $=0.020$ ) (Table 2 and Figure 2). On the contrary, HER2 gene amplification was more frequent in pure DCIS than in invasive carcinomas $(30.9 \%$ vs. $19.9 \%, P=0.004)$. $C-M Y C$ and $C C N D 1$ amplification did not differ between the two groups. Similarly there was no significant difference in the amplification frequencies of HER2, C$M Y C, C C N D 1$ and FGFR1 in pure DCIS and DCIS associated with invasive carcinomas (Table 2 and Figure 2).

In the next step, we compared gene amplification frequencies in DCIS and invasive carcinomas according to the grade of carcinoma (Table 2 and Figure 2), because DCIS are thought to be precursors of invasive cancers of similar grade. We found that HER2 and C-MYC amplification frequencies differed depending on the grade of carcinoma. In high-grade tumors, HER2 gene amplification frequency was also significantly higher in pure DICS than in invasive carcinoma $(59.7 \%$ vs. $34.4 \%, P<0.001)$. Conversely, the FGFR1 amplification frequency tended to be higher in invasive carcinoma than in pure DCIS (15.6\% vs. $6.8 \%, P=0.056$ ). HER2 amplification was more frequent in high-grade pure DCIS than in DCIS associated with invasive carcinoma $(59.7 \%$ vs. $38.2 \%, P=$ $0.004)$. For low/intermediate-grade tumors, however, there were no significant differences between the frequencies of gene amplification in pure DCIS, DCIS associated with invasive carcinomas, and invasive carcinomas.

We also evaluated co-amplification of genes and their associations in pure DCIS and invasive carcinoma (see Table S1 in Additional file 1). CCND1 amplification was correlated with HER2 and FGFR1 amplification in invasive carcinoma, but not in the pure DCIS.

\section{Comparison of gene amplification in the invasive and DCIS components of the same tumors}

To evaluate the role of gene amplification in the progression of DCIS to invasive carcinomas, we compared the gene amplification status of matched invasive and DCIS components in the 203 cases of invasive carcinomas with a DCIS component (Figure 3). The HER2, C-MYC, CCND1 and FGFR1 amplification status in the invasive and DCIS components agreed in most cases in the initial TMA examination. For the discrepant cases, we reanalyzed the gene amplification status of both components using all possible tumor sections with a DCIS component to find the potential area of gene amplification, and found that two of the discrepant cases in which only the invasive component showed gene amplification in initial TMA had a minor DCIS component with gene amplification in the whole section (Table S2 in Additional file 2).

The combined data for initial TMA and the whole-section examination revealed the concordance rate of $99.0 \%$ for HER2, $96.0 \%$ for $C-M Y C, 98.0 \%$ for $C C C N D 1$ and 96.9\% for FGFR1 amplification (Figure 3). HER2 was amplified in both the invasive and DCIS components in 46 cases $(22.8 \%)$, and in two cases $(1.0 \%)$ there was amplification only in the DCIS component. C-MYC was amplified in 18 cases (8.9\%) in both components, of which one case showed heterogeneous amplification in the DCIS component; that is, amplification was seen in 


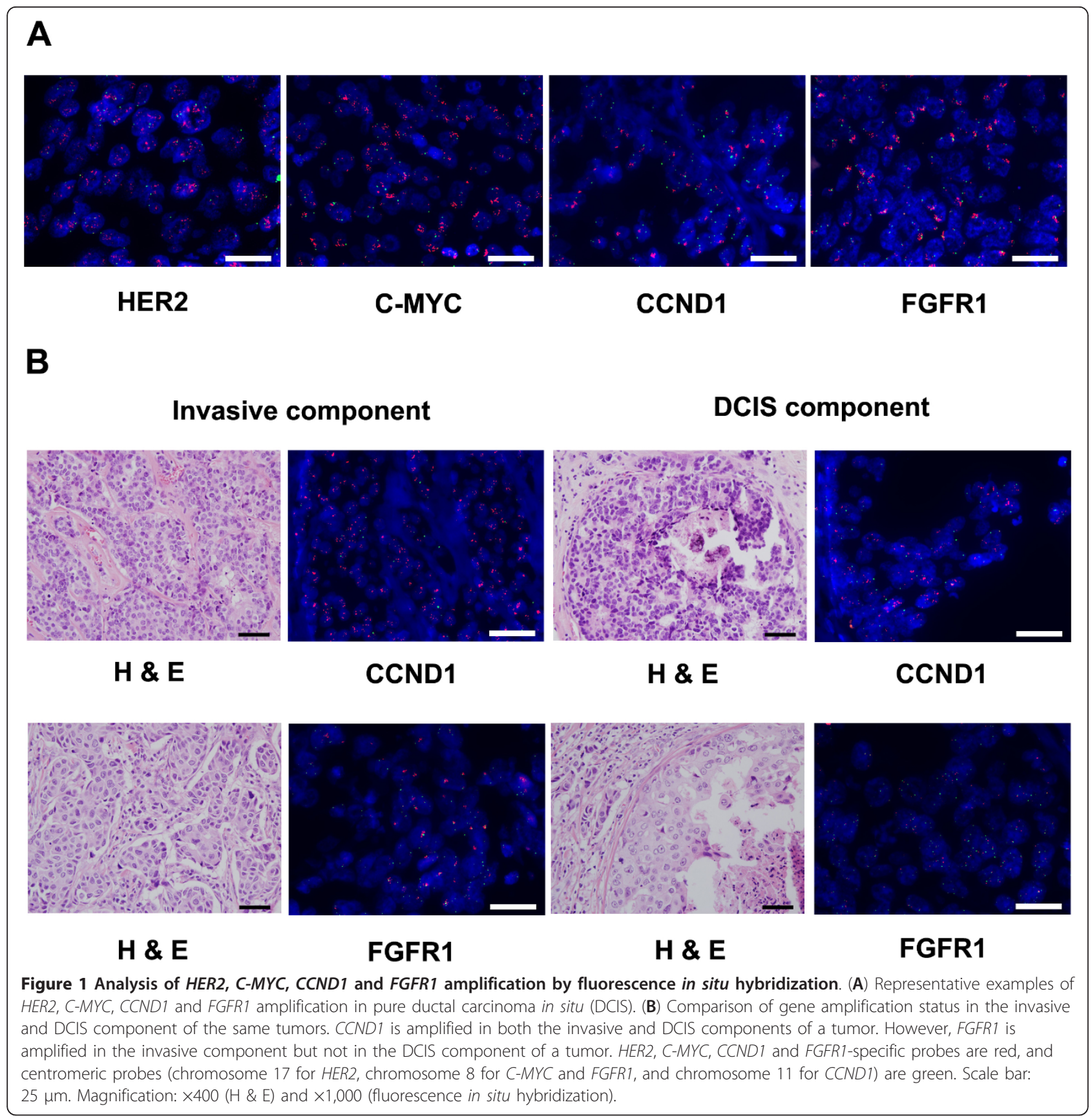

some DCIS ducts but not in others. C-MYC amplification was present only in the invasive component in six cases (3.0\%), in four of which amplification was variable. In two cases (1.0\%), only the DCIS component showed heterogeneous $C-M Y C$ amplification. CCND1 was amplified in both components in $32(15.9 \%)$ cases, in four of which amplification in the DCIS component was heterogeneous. CCND1 was amplified in only the DCIS component in three cases $(1.5 \%)$ and in only the invasive component in one case $(0.5 \%)$. Interestingly, amplification was heterogeneous in all four discordant cases. FGFR1 amplification was observed in both components in 20 cases (10.3\%), in three of which amplification in the DCIS component was variable. In six cases (3.1\%) only the invasive component showed FGFR1 amplification. FGFR1 amplification was thus found to be more frequent in the invasive component than in the DCIS component $(P=0.031)$.

Association of gene amplification with the clinicopathologic characteristics of tumor We also explored the associations between gene amplification and the clinicopathologic variables of the tumors 
Table 2 Gene amplification frequencies in invasive carcinoma, DCIS associated with invasive carcinoma, and pure DCIS

\begin{tabular}{|c|c|c|c|c|c|c|}
\hline Group & Gene & Pure DCIS & DCIS associated with invasive carcinoma & Invasive carcinoma & $P$ value $^{a}$ & $P$ value $^{\mathrm{b}}$ \\
\hline \multirow[t]{4}{*}{ Total } & HER2 & $54 / 175(30.9)$ & 48/202 (23.8) & 85/427 (19.9) & 0.004 & 0.122 \\
\hline & C-MYC & $17 / 173(9.8)$ & 20/203 (9.9) & $54 / 427(12.6)$ & 0.333 & 0.993 \\
\hline & CCND1 & 22/175 (12.6) & $35 / 201(17.4)$ & $61 / 424(14.4)$ & 0.559 & 0.192 \\
\hline & FGFR1 & $10 / 168(6.0)$ & 21/196 (10.7) & $52 / 417(12.5)$ & 0.020 & 0.105 \\
\hline \multirow[t]{4}{*}{ High grade } & HER2 & $46 / 77(59.7)$ & 39/102 (38.2) & 63/183 (34.4) & $<0.001$ & 0.004 \\
\hline & C-MYC & $12 / 76(15.8)$ & 15/103 (14.6) & $42 / 183(23.0)$ & 0.196 & 0.821 \\
\hline & CCND1 & 13/77 (16.9) & 23/102 (22.5) & $30 / 182(16.5)$ & 0.937 & 0.349 \\
\hline & FGFR1 & $5 / 74(6.8)$ & 14/97 (14.4) & 28/179 (15.6) & 0.056 & 0.114 \\
\hline \multirow[t]{4}{*}{ Low/intermediate grade } & HER2 & 8/98 (8.2) & 9/100 (9.0) & 22/230 (9.6) & 0.687 & 0.834 \\
\hline & C-MYC & $5 / 97(5.2)$ & 5/100 (5.0) & $11 / 230(4.8)$ & 1.000 & 1.000 \\
\hline & CCND1 & 9/98 (9.2) & 12/99 (12.1) & 29/228 (12.7) & 0.362 & 0.504 \\
\hline & FGFR1 & $5 / 94(5.3)$ & 7/99 (7.1) & 23/225 (10.2) & 0.158 & 0.614 \\
\hline
\end{tabular}

Data presented as $n$ (\%). $P$ values calculated using the chi-square test or Fisher's exact test. DCIS, ductal carcinoma in situ. ${ }^{\text {PPure }}$ DCIS versus invasive carcinoma;

${ }^{\mathrm{b}}$ Pure DCIS versus DCIS associated with invasive carcinoma.

(Table S3 in Additional file 3). In the invasive carcinomas, HER2 and $C-M Y C$ amplification were associated with the aggressive features of tumor, such as high histologic grade, ER/PR negativity, p53 overexpression and high Ki-67 proliferation index. CCND1 amplification was only correlated with ER positivity, and FGFR1 amplification was not associated with any clinicopatholgic features of the tumors. However, the association of FGFR1 amplification with high histologic grade was close to significance $(P=0.095)$. In pure DCIS, HER2

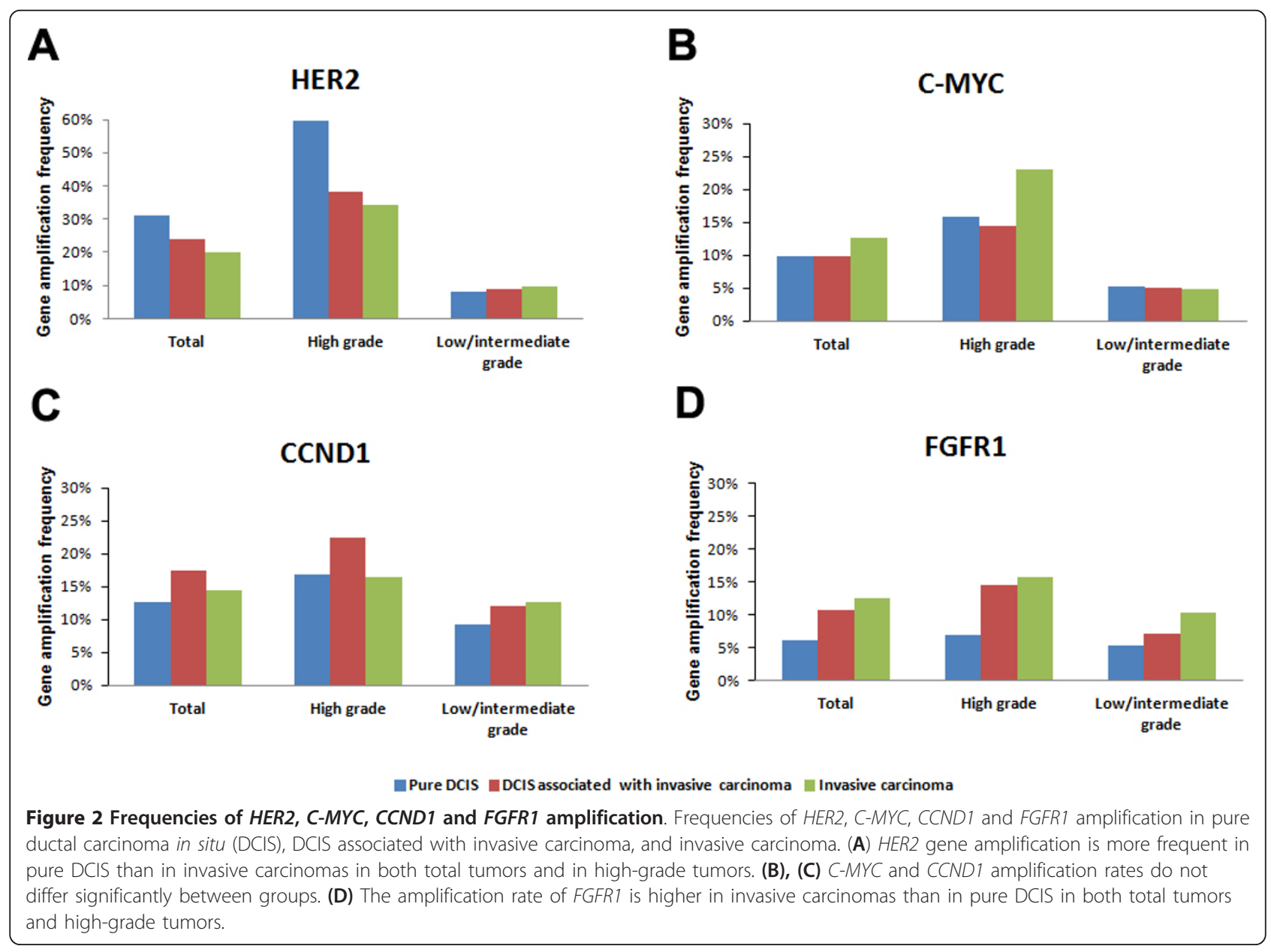




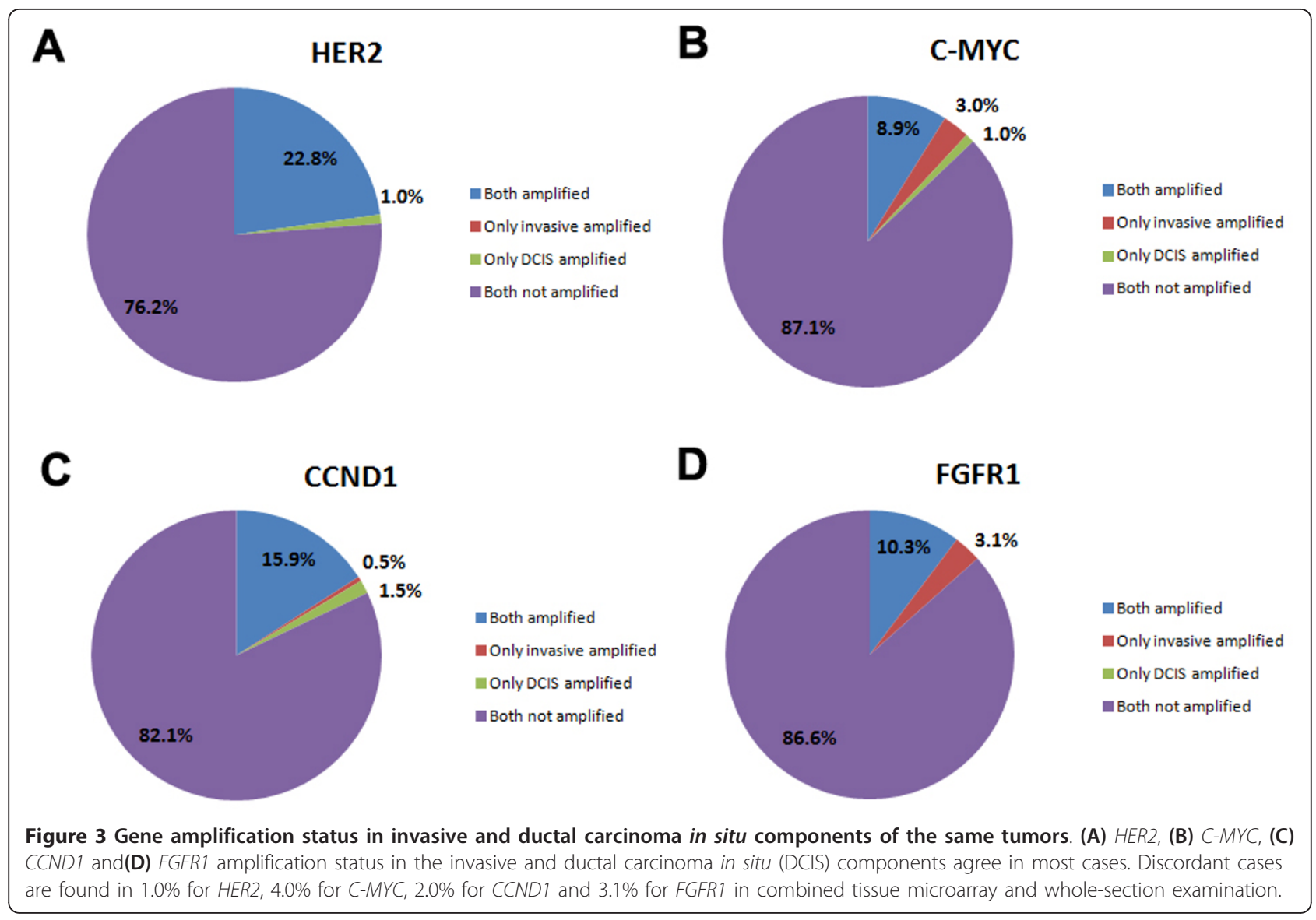

and $C-M Y C$ amplification were also associated with high nuclear grade and high Ki-67 proliferation index. However, no clinicopathologic variables of DCIS were associated with FGFR1 amplification.

Gene amplification frequencies were also associated with the tumor subtype (Table 3 ). Specifically, CCND1 amplification was not found in any of the progression stages of basal-like breast cancer. In invasive carcinoma, $C-M Y C$ amplification was most common in basal-like subtype and was more frequent in the luminal B subtype than in the luminal A subtype. CCND1 and FGFR1 amplification was most common in the luminal B subtype, being significantly higher than in the luminal A, HER2-positive and triple-negative, nonbasal subtypes. In pure DCIS, CCND1 and FGFR1 amplification was also most common in the luminal B subtype and tended to be more frequent in the luminal $B$ subtype than in the luminal A subtype $(P=0.099$ and $P=0.071$, respectively). However, there were no significant differences in gene amplification frequencies between pure DCIS and invasive carcinoma within the individual subtypes, although FGFR1 amplification frequencies were higher (although not significantly so) in the invasive carcinomas than in the pure DCIS in all subtypes except the triplenegative, nonbasal subtype.

\section{FGFR1 amplification as an independent prognostic factor for breast cancer progression}

We also investigated the prognostic role of gene amplification in invasive breast cancer. Most patients were treated by the standard practice guidelines and have been followed regularly after surgery. Among the 85 patients with HER2-amplified breast cancer, 20 patients (24\%) received adjuvant trastuzumab therapy. There were five patients with initial metastases at the time of operation, and 422 patients were analyzed for disease-free survival. The median follow-up time was 5 years (range 1 to 8 years). There were 10 (2.4\%) loco-regional recurrences and $34(8.1 \%)$ distant metastases as first events. In Kaplan-Meier survival analyses, the patients with FGFR1amplified breast cancer had shorter disease-free survival times than those without it $(P=0.003$; Figure $4 \mathrm{~A})$. On the contrary, there were no survival differences associated with HER-2, C-MYC or CCND1 amplification $(P=0.160$, $P=0.268$, and $P=0.670$, respectively). Subgroup analyses also revealed a difference in survival between the patients 
Table 3 Relationships between gene amplification and molecular subtypes

\begin{tabular}{|c|c|c|c|c|c|c|c|}
\hline \multirow[b]{2}{*}{ Histologic stage } & \multirow[b]{2}{*}{ Gene } & \multicolumn{6}{|l|}{ Subtype } \\
\hline & & Luminal A & Luminal B & HER2-positive & Basal-like & TNNB & $P$ value $^{\mathrm{a}}$ \\
\hline \multirow[t]{3}{*}{ Invasive carcinoma } & C-MYC & $13 / 208(6.3)^{* * *}$ & $15 / 103(14.6)^{* *}$ & $7 / 42(16.7)$ & $14 / 55(25.5)^{*}$ & $5 / 19(26.3)$ & $<0.001$ \\
\hline & CCND1 & $19 / 205(9.3)^{\dagger}$ & $37 / 103(35.9)^{t, \neq, \S}$ & $4 / 42(9.5)^{\ddagger}$ & $0 / 55(0)^{\dagger}$ & $1 / 18(5.3)^{\S}$ & $<0.001$ \\
\hline & FGFR1 & $21 / 202(10.4)^{\pi}$ & $21 / 100(21.0)^{\mathbf{9},+t, \ldots \neq}$ & $3 / 42(7.1)^{t+}$ & $7 / 55(12.7)$ & $0 / 18(0)^{\neq \neq}$ & 0.025 \\
\hline \multirow[t]{3}{*}{ Pure DCIS } & C-MYC & $6 / 98(6.1)$ & $4 / 24(16.7)$ & 6/34 (17.6) & $1 / 9(11.1)$ & $0 / 8(0)$ & 0.198 \\
\hline & CCND1 & 11/99 (11.1) & $6 / 24(25.0)$ & 4/35 (11.4) & 0/9 (0) & $1 / 8(12.5)$ & 0.298 \\
\hline & FGFR1 & $5 / 95(5.3)$ & $4 / 23(17.4)$ & $1 / 33(3.0)$ & $0 / 9(0)$ & $0 / 8(0)$ & 0.134 \\
\hline
\end{tabular}

Data presented as $n$ (\%). $P$ values calculated using the chi-square test or Fisher's exact test. DCIS, ductal carcinoma in situ; TNNB, triple negative, nonbasal. ${ }^{a}$ Between molecular subtypes. ${ }^{*} P<0.001$, basal-like vs. luminal $A$. ${ }^{* *} P=0.016$, luminal $B$ vs. luminal $A$ for $C-M Y C$. ${ }^{\dagger} P<0.001$, luminal $B$ vs. luminal $A$, luminal $B$ vs. basal-like. ${ }^{\ddagger} P=0.001$, luminal B vs. HER2-positive. ${ }^{\S} P=0.008$, luminal B vs. TNNB for $C C N D 1$. ${ }^{\Uparrow} P=0.012$, luminal $B$ vs. luminal $A$. ${ }^{+\dagger} P=0.044$, luminal $B$ vs. HER2positive. ${ }^{\ddagger \ddagger} P=0.040$, luminal B vs. TNNB for FGFR1.

with and without FGFR1-amplified breast cancer in the hormone receptor-positive group $(P=0.001$; Figure $4 B)$, but not in the hormone receptor-negative group $(P=$ 0.284 ; Figure $4 C$ ). Similarly, there was no survival differences with regard to FGFR1 amplification in the HER2positive subtype $(P=0.514)$ and the basal-like subtype $(P=0.505)$.

In addition to FGFR 1 amplification, high $\mathrm{T}$ stage (T1 to T2 vs. T3 to T4, $P<0.001$ ), nodal metastasis (N0 vs. N1 to N3, $P=0.002)$, hormone receptor negativity $(P=$ 0.004), p53 overexpression $(P=0.005)$ and high Ki-67 proliferation index $(P=0.006)$ were significantly associated with poor disease-free survival in all patients with invasive carcinoma. In multivariate analysis, only the T stage (pT1 to pT2 vs. pT3 to pT4, $\mathrm{HR}=2.916,95 \% \mathrm{CI}=1.254$ to $6.781, P=0.013$ ), N stage (N0 vs. N1 to N3, HR $=2.585$, $95 \% \mathrm{CI}=1.324$ to $5.049, P=0.005)$ and $F G F R 1$ amplification $(\mathrm{HR}=2.794,95 \% \mathrm{CI}=1.375$ to $5.678, P=0.001)$ remained independent prognostic factors for disease-free survival. In the hormone receptor-positive group, the $\mathrm{N}$ stage (N0 vs. $\mathrm{N} 1$ to $\mathrm{N} 3, \mathrm{HR}=4.514,95 \% \mathrm{CI}=1.506$ to 13.531, $P=0.007)$, p53 overexpression $(\mathrm{HR}=2.757,95 \%$ $\mathrm{CI}=1.029$ to $7.388, P=0.044)$ and FGFR1 amplification $(\mathrm{HR}=2.659,95 \% \mathrm{CI}=1.042$ to $6.785, P=0.041)$ were identified as independent prognostic factors.

\section{Discussion}

In this study we have shown that FGFR1 amplification is more frequent in invasive carcinoma than in pure DCIS, and in the invasive components of tumors with invasive and DICS components. In addition, FGFR1 amplification was found to be associated with decreased disease-free survival, suggesting a role for FGFR1 amplification in the progression of breast cancer including the in situ to invasive transition.

FGFR1 was suggested to be the target oncogene implicated in 8p11-12 amplification [15,35,36]. However, Gelsi-Boyer and colleagues reported that the 8p11-12 amplicon is much more complex, and is composed of at least four amplicons including 14 candidate oncogenes

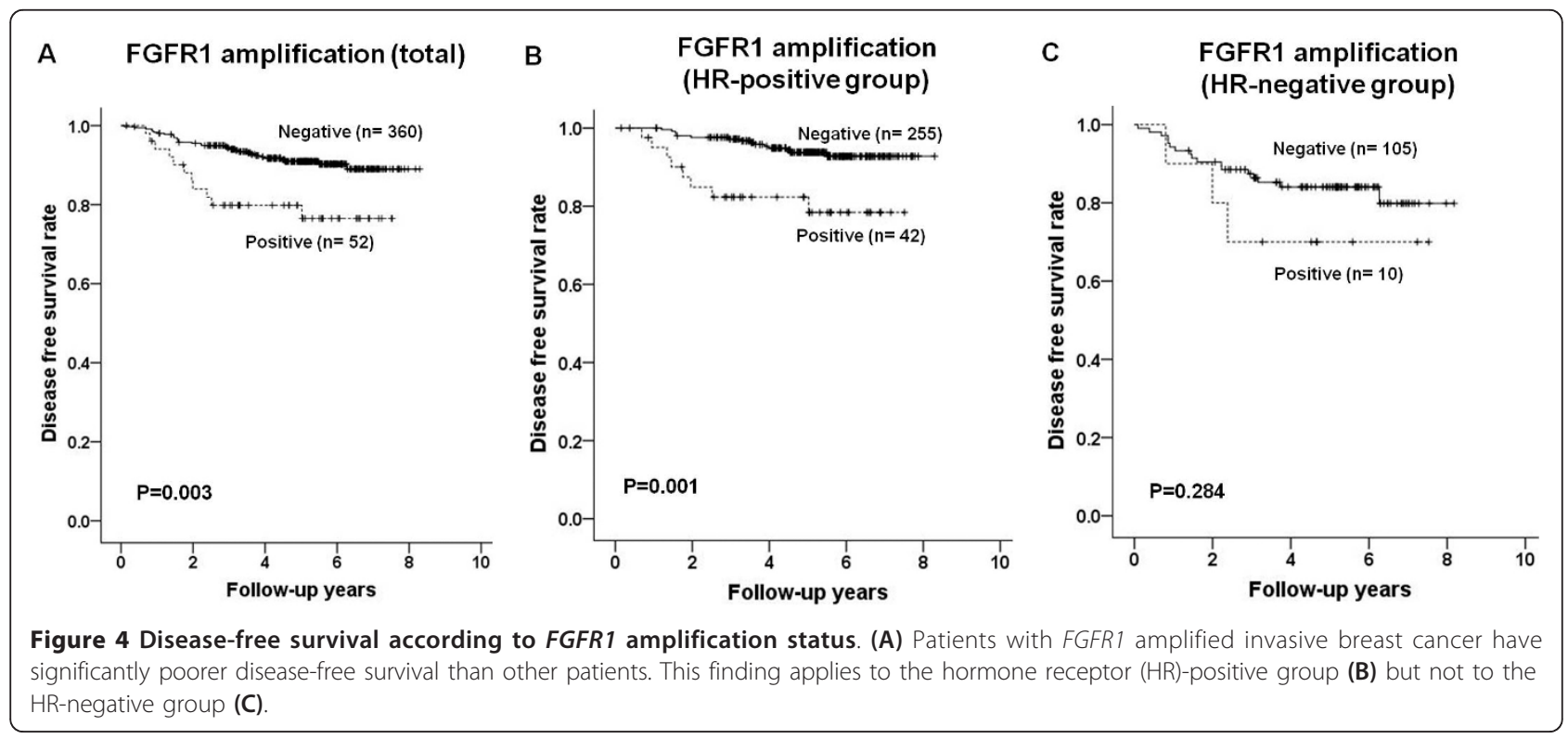


that can be amplified independently [37]. Not only FGFR1 but also other oncogenes in the 8p11-12 amplicon, such as LSM1, BAG4 and C8orf4, are therefore now accepted as contributing to oncogenesis [38,39]. Although FGFR1 is not widely accepted as the driver breast cancer oncogene affected in the 8p11-12 amplification, its amplification has been reported to be associated with poor prognosis, especially in patients with ER-positive tumors [14]. In this study, we confirmed the prognostic impact of FGFR 1 amplification, especially in the hormone receptor-positive group. Furthermore, we showed that FGFR1 amplification is most frequently found in the luminal B subtype among the various breast cancer subtypes. No correlations were found between FGFR1 amplification and clinicopathologic features of the tumors. In the hormone receptor-positive group, however, FGFR1 amplification was associated with high histologic grade $(P=0.018)$, PR negativity $(P=0.017)$ and high Ki-67 proliferation index $(P=0.020)$ (data not shown). Recently, in accord with our findings, Turner and colleagues reported that FGFR1 signaling suppressed PR expression and that FGFR1-amplified cancers had higher Ki-67 proliferation indices and were most often found in the luminal B subtype, accounting for 16 to $27 \%$ of their series [17]. They also demonstrated that FGFR1 amplification increased resistance to endocrine therapy. Taken together, these findings suggest that FGFR1 amplification makes an important contribution to the aggressive phenotype of hormone receptor-positive breast cancer.

Although FGFR1 amplification has been shown to be associated with breast cancer progression, there have been no studies of its association with the in situ to invasive transition. In our study, FGFR1 amplification was more frequent in invasive carcinoma than in pure DCIS, and tended to be higher in invasive carcinomas than in pure DCIS in the high-grade tumors. More importantly, FGFR1 amplification was more frequent in the invasive components of tumors than in the corresponding DCIS components. The mechanism by which FGFR1 amplification induces invasion of DCIS is unknown. However, in the mouse mammary tumor virus-inducible FGFR1 transgenic mouse model, sustained activation of FGFR1 in the mouse mammary epithelium induces alveolar hyperplasia and invasive lesions, which are associated with extracellular matrix remodeling and vascular branching in the stroma adjacent to these lesions [40]. The same authors have developed an in vitro threedimensional HC11 mouse mammary epithelial cell culture model expressing a drug-inducible FGFR1 and have demonstrated that inducible FGFR1 activation results in a gain of invasive properties and promotes the epithelialmesenchymal transition, which is caused by induction of matrix metalloproteinase-3 [41]. FGFR1 amplification, and hence increased FGFR1 signaling, therefore seems to contribute to early breast cancer invasion; that is, the in situ to invasive transition. This result may have clinical implications, because DCIS with FGFR1 amplification is more likely to progress to invasive carcinoma. In this study, we focused on the role of genetic alterations in the progression of DCIS to invasive carcinoma. However, a growing body of evidence suggests that the transition of DCIS to invasive carcinoma is strongly dependent on the tumor microenvironment, particularly on the myoepithelial cells and cancer-associated fibroblasts $[42,43]$. Further studies will therefore be needed to elucidate the effect of FGFR1 amplification in epithelial cells on the tumor microenvironment and epithelial-stromal interactions.

We found no differences in the amplification frequencies of C-MYC and CCND1 between DCIS, DCIS associated with invasive carcinoma, and invasive carcinoma. Burkhardt and colleagues recently examined the amplification frequencies of HER2, ESR1, CCND1 and MYC in a large series of pure DCIS and DCIS associated with invasive carcinomas, and also found no significant differences between them [28]. However, we obtained different results for HER2 gene amplification. This amplification was significantly more frequent in pure DCIS than in invasive carcinomas, especially in high-grade tumors, and other workers have obtained the same result $[44,45]$. Amplification of HER2, C-MYC and CCND1 therefore seems to play a role in the early development of breast cancer, but not in the progression of DCIS to invasive carcinomas.

In a comparison of the matched DCIS and invasive components of the same tumors we demonstrated that the genetic changes in the two components were similar in terms of HER2, C-MYC and CCND1 gene amplification. However, we detected amplification in the DCIS components but not in the invasive carcinoma components in a few cases for HER2 $(n=2), C-M Y C(n=2)$ and CCND1 $(n=3)$. Interestingly, the two cases with $C-M Y C$ amplification and the three cases with $C C N D 1$ amplification in only the DCIS component showed heterogeneous amplification. Four of the 32 cases with CCND1 amplification in both components and three of the 20 cases that had FGFR1 amplification in both components also had heterogeneous amplification in the DCIS component. Furthermore, in pure DCIS, heterogeneity of gene amplification was found in $3.7 \%$ (2/52) for HER2, $11.8 \%(2 / 17)$ for $C$ $M Y C, 22.7 \%$ (5/22) for CCND1 and 10\% (1/10) for FGFR1 amplified cases (data not shown). These findings suggest that intra-tumoral genetic heterogeneity is already present in the DCIS and that progression of DCIS to invasive carcinomas may result from selection of subpopulations of tumor cells. In a previous study we reported that the differences in molecular subtypes among the invasive tumor 
foci of multifocal/multicentric breast cancers were associated with mixed molecular subtypes in the DCIS components, suggesting that heterogeneity within the DCIS followed by selection of different clones might give rise to the different phenotypes in multicentric/multifocal breast cancers [46]. Supporting this concept, Hernandez and colleagues recently performed comparative analyses of known cancer genes using microarray-based comparative genomic hybridization and Sequenom MassARRAY (OncoCarta Panel v 1.0, Sequenom, San Diego, CA, USA) in matched DCIS and adjacent invasive carcinomas, and suggested that although the modal populations of both components were similar at the genetic level, the progression from DCIS to invasive ductal carcinoma was driven in some cases by selection of nonmodal clones with specific genetic aberrations [47].

The different breast cancer subtypes have been suggested to have distinct patterns of copy number alterations. While higher numbers of gains/losses have been associated with the basal-like subtype, high-level DNA amplification has been found in the luminal B and HER2positive subtypes [48], and candidate oncogenes have been identified in chromosomal regions 1q21-23, 10p14 and $12 \mathrm{p} 13$ for basal-like breast cancers, but in regions 1q2123, 8p12-q21, 11q13 and 16p12-13 for luminal breast cancers [4]. Moreover, CCND1 amplification on 11q13 has been reported to be associated with luminal subtypes [16], and in agreement with this we found that CCND1 and FGFR1 amplification was most frequent in the luminal B subtype whereas CCND1 amplification was absent from all of the basal-like breast cancers.

\section{Conclusion}

In summary, we have studied the amplification frequencies of HER2, C-MYC, CCND1 and FGFR1 in a large series of pure DCIS, DCIS associated with invasive carcinoma, and invasive carcinomas, to investigate the role of gene amplification in the progression of DCIS to invasive carcinoma. The amplification frequencies of $C-M Y C$ and CCND1 did not differ between pure DCIS and invasive carcinomas, and HER2 amplification was more frequent in pure DCIS. The HER2, C-MYC, CCND1 and FGFR1 amplification status was in most cases concordant in the matched invasive and DCIS components of the same tumors, pointing to early roles in the development of breast cancer. However, FGFR1 amplification was more frequent in invasive carcinomas than in pure DCIS, and in the invasive components of the same tumors. Furthermore, FGFR1 amplification was found to be an independent prognostic factor for disease-free survival. Our results therefore suggest that FGFR1 amplification play an important role in the progression of breast cancer, including the in situ to invasive transition, as well as initiation.

\section{Additional material}

\section{Additional file 1: Table S1 presenting frequencies of co-}

amplification of genes (A) and their correlations (B) in invasive carcinoma and pure DCIS.

Additional file 2: Table S2 presenting a comparison of initial TMA and whole-section examination for gene amplification status in discrepant cases for invasive and in situ components of a same tumor.

Additional file 3: Table S3 presenting correlation of HER2, C-MYC, CCND1 and FGFR1 amplification with clinicopathologic characteristics in invasive (A) and in situ (B) breast carcinomas.

\section{Abbreviations}

CEP: chromosome enumeration probe; Cl: confidence interval; DCIS: ductal carcinoma in situ; EGFR: epidermal growth factor receptor; ER: estrogen receptor; FGFR1: fibroblast growth factor receptor 1; FISH: fluorescence in situ hybridization; H \& E: hematoxylin and eosin; HER2: human epidermal growth factor receptor 2; HR: hazard ratio; LSI: locus specific identifier; PR: progesterone receptor; TMA: tissue microarray.

\section{Acknowledgements}

This research was supported by the Basic Science Research Program through the National Research Foundation of Korea (NRF) funded by the Ministry of Education, Science and Technology (Grant No. 2011-0005483).

\section{Author details}

${ }^{1}$ Department of Pathology, Seoul National University College of Medicine, 103 Daehakro, Jongno-gu, Seoul 110-799, Korea. ${ }^{2}$ Department of Pathology, Seoul National University Hospital, 101 Daehakro, Jongno-gu, Seoul 110-744, Korea. ${ }^{3}$ Department of Pathology, Seoul National University Bundang Hospital, 300 Gumi-dong, Bundang-gu, Seongnam, Gyeonggi 463-707, Korea. ${ }^{4}$ Breast Care Center, Seoul National University Bundang Hospital, 300 Gumidong, Bundang-gu, Seongnam, Gyeonggi 463-707, Korea.

\section{Authors' contributions}

$\mathrm{MHJ}$ participated in the interpretation and analysis of data and drafted the manuscript. EJK carried out the experiments and participated in the interpretation of the data. YC and HEL participated in the acquisition and interpretation of pathologic data. YJK, JHK, EK, S-WK and IAK participated in the acquisition of clinical data. SYP conceived of the study, and participated in its design and was responsible for preparation of the manuscript. All authors read and approved the final manuscript.

\section{Competing interests}

The authors declare that they have no competing interests.

Received: 10 May 2012 Revised: 5 July 2012 Accepted: 3 August 2012 Published: 3 August 2012

\section{References}

1. Beckmann MW, Niederacher D, Schnurch HG, Gusterson BA, Bender HG. Multistep carcinogenesis of breast cancer and tumour heterogeneity. J Mol Med (Berl) 1997, 75:429-439.

2. Courjal F, Theillet C: Comparative genomic hybridization analysis of breast tumors with predetermined profiles of DNA amplification. Cancer Res 1997, 57:4368-4377

3. Yao J, Weremowicz S, Feng B, Gentleman RC, Marks JR, Gelman R, Brennan C, Polyak K: Combined cDNA array comparative genomic hybridization and serial analysis of gene expression analysis of breast tumor progression. Cancer Res 2006, 66:4065-4078.

4. Adelaide J, Finetti P, Bekhouche I, Repellini L, Geneix J, Sircoulomb F, Charafe-Jauffret E, Cervera N, Desplans J, Parzy D, Schoenmakers E, Viens P, Jacquemier J, Birnbaum D, Bertucci F, Chaffanet M: Integrated profiling of basal and luminal breast cancers. Cancer Res 2007, 67:11565-11575.

5. Nikolsky Y, Sviridov E, Yao J, Dosymbekov D, Ustyansky V, Kaznacheev V, Dezso Z, Mulvey L, Macconaill LE, Winckler W, Serebryiskaya T, Nikolskaya T, 
Polyak K: Genome-wide functional synergy between amplified and mutated genes in human breast cancer. Cancer Res 2008, 68:9532-9540.

6. Press MF, Pike MC, Chazin VR, Hung G, Udove JA, Markowicz M, Danyluk J, Godolphin W, Sliwkowski M, Akita R, Paterson MC, Slamon DJ: Her-2/neu expression in node-negative breast cancer: direct tissue quantitation by computerized image analysis and association of overexpression with increased risk of recurrent disease. Cancer Res 1993, 53:4960-4970.

7. Slamon DJ, Clark GM, Wong SG, Levin WJ, Ullrich A, McGuire WL: Human breast cancer: correlation of relapse and survival with amplification of the HER-2/neu oncogene. Science 1987, 235:177-182.

8. Xu J, Chen Y, Olopade Ol: MYC and breast cancer. Genes Cancer 2010, 1:629-640

9. Deming SL, Nass SJ, Dickson RB, Trock BJ: C-myc amplification in breast cancer: a meta-analysis of its occurrence and prognostic relevance. $\mathrm{Br} J$ Cancer 2000, 83:1688-1695.

10. Schlotter CM, Vogt U, Bosse U, Mersch B, Wassmann K: C-myc, not HER-2/ neu, can predict recurrence and mortality of patients with nodenegative breast cancer. Breast Cancer Res 2003, 5:R30-R36.

11. Aulmann S, Adler N, Rom J, Helmchen B, Schirmacher P, Sinn HP: c-myc amplifications in primary breast carcinomas and their local recurrences. J Clin Pathol 2006, 59:424-428.

12. Rodriguez-Pinilla SM, Jones RL, Lambros MB, Arriola E, Savage $K$, James M, Pinder SE, Reis-Filho JS: MYC amplification in breast cancer: a chromogenic in situ hybridisation study. J Clin Pathol 2007, 60:1017-1023.

13. Eswarakumar VP, Lax I, Schlessinger J: Cellular signaling by fibroblast growth factor receptors. Cytokine Growth Factor Rev 2005, 16:139-149.

14. Elbauomy Elsheikh S, Green AR, Lambros MB, Turner NC, Grainge MJ, Powe D, Ellis 1O, Reis-Filho JS: FGFR1 amplification in breast carcinomas: a chromogenic in situ hybridisation analysis. Breast Cancer Res 2007, 9:R23.

15. Courjal F, Cuny M, Simony-Lafontaine J, Louason G, Speiser P, Zeillinger R, Rodriguez C, Theillet C: Mapping of DNA amplifications at 15 chromosomal localizations in 1875 breast tumors: definition of phenotypic groups. Cancer Res 1997, 57:4360-4367.

16. Letessier A, Sircoulomb F, Ginestier C, Cervera N, Monville F, Gelsi-Boyer V, Esterni B, Geneix J, Finetti P, Zemmour C, Viens P, Charafe-Jauffret $E$, Jacquemier J, Birnbaum D, Chaffanet M: Frequency, prognostic impact, and subtype association of $8 p 12,8 q 24,11 q 13,12 p 13,17 q 12$, and $20 q 13$ amplifications in breast cancers. BMC Cancer 2006, 6:245.

17. Turner N, Pearson A, Sharpe R, Lambros M, Geyer F, Lopez-Garcia MA, Natrajan R, Marchio C, lorns E, Mackay A, Gillett C, Grigoriadis A, Tutt A, Reis-Filho JS, Ashworth A: FGFR1 amplification drives endocrine therapy resistance and is a therapeutic target in breast cancer. Cancer Res 2010, 70:2085-2094.

18. Baldin V, Lukas J, Marcote MJ, Pagano M, Draetta G: Cyclin D1 is a nuclear protein required for cell cycle progression in G1. Genes Dev 1993, 7:812-821

19. Ormandy CJ, Musgrove EA, Hui R, Daly RJ, Sutherland RL: Cyclin D1, EMS1 and 11q13 amplification in breast cancer. Breast Cancer Res Treat 2003, 78:323-335.

20. Erbas B, Provenzano E, Armes J, Gertig D: The natural history of ductal carcinoma in situ of the breast: a review. Breast Cancer Res Treat 2006, 97:135-144.

21. Allred DC, Wu Y, Mao S, Nagtegaal ID, Lee S, Perou CM, Mohsin SK, O'Connell P, Tsimelzon A, Medina D: Ductal carcinoma in situ and the emergence of diversity during breast cancer evolution. Clin Cancer Res 2008, 14:370-378.

22. Vincent-Salomon A, Lucchesi C, Gruel N, Raynal V, Pierron G, Goudefroye R, Reyal F, Radvanyi F, Salmon R, Thiery JP, Sastre-Garau X, Sigal-Zafrani B, Fourquet A, Delattre O: Integrated genomic and transcriptomic analysis of ductal carcinoma in situ of the breast. Clin Cancer Res 2008, 14:1956-1965.

23. Buerger $H$, Otterbach $F$, Simon R, Poremba C, Diallo R, Decker $T$, Riethdorf $L$, Brinkschmidt C, Dockhorn-Dworniczak B, Boecker W: Comparative genomic hybridization of ductal carcinoma in situ of the breast-evidence of multiple genetic pathways. J Pathol 1999, 187:396-402.

24. Vos CB, ter Haar NT, Rosenberg C, Peterse UL, Cleton-Jansen AM, Cornelisse CJ, van de Vijver MJ: Genetic alterations on chromosome 16 and 17 are important features of ductal carcinoma in situ of the breast and are associated with histologic type. Br J Cancer 1999, 81:1410-1418.
25. Hwang ES, DeVries S, Chew KL, Moore DH, Kerlikowske K, Thor A, Ljung BM, Waldman FM: Patterns of chromosomal alterations in breast ductal carcinoma in situ. Clin Cancer Res 2004, 10:5160-5167.

26. Ma XJ, Salunga R, Tuggle JT, Gaudet J, Enright E, McQuary P, Payette T, Pistone M, Stecker K, Zhang BM, Zhou YX, Varnholt H, Smith B, Gadd M, Chatfield E, Kessler J, Baer TM, Erlander MG, Sgroi DC: Gene expression profiles of human breast cancer progression. Proc Natl Acad Sci USA 2003, 100:5974-5979.

27. Glockner S, Lehmann U, Wilke N, Kleeberger W, Langer F, Kreipe H: Amplification of growth regulatory genes in intraductal breast cancer is associated with higher nuclear grade but not with the progression to invasiveness. Lab Invest 2001, 81:565-571.

28. Burkhardt L, Grob TJ, Hermann I, Burandt E, Choschzick M, Janicke F, Muller V, Bokemeyer C, Simon R, Sauter G, Wilczak W, Lebeau A: Gene amplification in ductal carcinoma in situ of the breast. Breast Cancer Res Treat 2010, 123:757-765.

29. Moelans CB, de Weger RA, Monsuur HN, Maes AH, van Diest PJ: Molecular differences between ductal carcinoma in situ and adjacent invasive breast carcinoma: a multiplex ligation-dependent probe amplification study. Anal Cell Pathol (Amst) 2010, 33:165-173.

30. Robanus-Maandag EC, Bosch CA, Kristel PM, Hart AA, Faneyte IF, Nederlof PM, Peterse JL, van de Vijver MJ: Association of C-MYC amplification with progression from the in situ to the invasive stage in C-MYC-amplified breast carcinomas. J Pathol 2003, 201:75-82.

31. Corzo C, Corominas JM, Tusquets I, Salido M, Bellet M, Fabregat X Serrano S, Sole F: The MYC oncogene in breast cancer progression: from benign epithelium to invasive carcinoma. Cancer Genet Cytogenet 2006, 165:151-156

32. Hyun CL, Lee HE, Kim KS, Kim SW, Kim JH, Choe G, Park SY: The effect of chromosome 17 polysomy on HER-2/neu status in breast cancer. J Clin Pathol 2008, 61:317-321.

33. Hammond ME, Hayes DF, Dowsett M, Allred DC, Hagerty KL, Badve S, Fitzgibbons PL, Francis G, Goldstein NS, Hayes M, Hicks DG, Lester S, Love R, Mangu PB, McShane L, Miller K, Osborne CK, Paik S, Perlmutter J, Rhodes A, Sasano H, Schwartz JN, Sweep FC, Taube S, Torlakovic EE, Valenstein P, Viale G, Visscher D, Wheeler T, Williams RB, et al: American Society of Clinical Oncology/College of American Pathologists guideline recommendations for immunohistochemical testing of estrogen and progesterone receptors in breast cancer. J Clin Oncol 2010, 28:2784-2795.

34. Voduc KD, Cheang MC, Tyldesley S, Gelmon K, Nielsen TO, Kennecke H: Breast cancer subtypes and the risk of local and regional relapse. J Clin Oncol 2010, 28:1684-1691.

35. Theillet C, Adelaide J, Louason G, Bonnet-Dorion F, Jacquemier J, Adnane J, Longy M, Katsaros D, Sismondi P, Gaudray P, Birnbaum D: FGFRI and PLAT genes and DNA amplification at 8p12 in breast and ovarian cancers. Genes Chromosomes Cancer 1993, 7:219-226.

36. Ugolini F, Adelaide J, Charafe-Jauffret E, Nguyen C, Jacquemier J, Jordan B, Birnbaum D, Pebusque MJ: Differential expression assay of chromosome arm 8p genes identifies Frizzled-related (FRP1/FRZB) and fibroblast growth factor receptor 1 (FGFR1) as candidate breast cancer genes. Oncogene 1999, 18:1903-1910.

37. Gelsi-Boyer V, Orsetti B, Cervera N, Finetti P, Sircoulomb F, Rouge C, Lasorsa L, Letessier A, Ginestier C, Monville F, Esteyries S, Adelaide J, Esterni B, Henry C, Ethier SP, Bibeau F, Mozziconacci MJ, Charafe-Jauffret E, Jacquemier J, Bertucci F, Birnbaum D, Theillet C, Chaffanet M: Comprehensive profiling of 8p11-12 amplification in breast cancer. $\mathrm{Mol}$ Cancer Res 2005, 3:655-667.

38. Yang ZQ, Streicher KL, Ray ME, Abrams J, Ethier SP: Multiple interacting oncogenes on the 8p11-p12 amplicon in human breast cancer. Cancer Res 2006, 66:11632-11643.

39. Streicher $\mathrm{KL}$, Yang ZQ, Draghici S, Ethier SP: Transforming function of the LSM1 oncogene in human breast cancers with the 8p11-12 amplicon. Oncogene 2007, 26:2104-2114.

40. Welm BE, Freeman KW, Chen M, Contreras A, Spencer DM, Rosen JM: Inducible dimerization of FGFR1: development of a mouse model to analyze progressive transformation of the mammary gland. J Cell Biol 2002, 157:703-714.

41. Xian W, Schwertfeger KL, Vargo-Gogola T, Rosen JM: Pleiotropic effects of FGFR1 on cell proliferation, survival, and migration in a 3D mammary epithelial cell model. J Cell Biol 2005, 171:663-673. 
42. Hu M, Yao J, Carroll DK, Weremowicz S, Chen H, Carrasco D, Richardson A, Violette S, Nikolskaya T, Nikolsky Y, Bauerlein EL, Hahn WC, Gelman RS, Allred C, Bissell MJ, Schnitt S, Polyak K: Regulation of in situ to invasive breast carcinoma transition. Cancer Cell 2008, 13:394-406.

43. Place $A E$, Jin Huh $S$, Polyak $K$ : The microenvironment in breast cancer progression: biology and implications for treatment. Breast Cancer Res 2011, 13:227.

44. Latta EK, Tjan S, Parkes RK, O'Malley FP: The role of HER2/neu overexpression/amplification in the progression of ductal carcinoma in situ to invasive carcinoma of the breast. Mod Pathol 2002, 15:1318-1325.

45. Park K, Han S, Kim HJ, Kim J, Shin E: HER2 status in pure ductal carcinoma in situ and in the intraductal and invasive components of invasive ductal carcinoma determined by fluorescence in situ hybridization and immunohistochemistry. Histopathology 2006, 48:702-707.

46. Choi Y, Kim EJ, Seol H, Lee HE, Jang MJ, Kim SM, Kim JH, Kim SW, Choe G, Park SY: The hormone receptor, human epidermal growth factor receptor 2, and molecular subtype status of individual tumor foci in multifocal/multicentric invasive ductal carcinoma of breast. Hum Pathol 2012, 43:48-55.

47. Hernandez L, Wilkerson PM, Lambros MB, Campion-Flora A, Rodrigues DN, Gauthier A, Cabral C, Pawar V, Mackay A, A'Hern R, Marchio C, Palacios J, Natrajan $R$, Weigelt B, Reis-Filho JS: Genomic and mutational profiling of ductal carcinomas in situ and matched adjacent invasive breast cancers reveals intra-tumour genetic heterogeneity and clonal selection. J Pathol 2012, 227:42-52.

48. Bergamaschi A, Kim YH, Wang P, Sorlie T, Hernandez-Boussard T, Lonning PE, Tibshirani R, Borresen-Dale AL, Pollack JR: Distinct patterns of DNA copy number alteration are associated with different clinicopathological features and gene-expression subtypes of breast cancer. Genes Chromosomes Cancer 2006, 45:1033-1040.

doi:10.1186/bcr3239

Cite this article as: Jang et al: FGFR1 is amplified during the progression of in situ to invasive breast carcinoma. Breast Cancer Research 2012 14:R115.

\section{Submit your next manuscript to BioMed Central and take full advantage of:}

- Convenient online submission

- Thorough peer review

- No space constraints or color figure charges

- Immediate publication on acceptance

- Inclusion in PubMed, CAS, Scopus and Google Scholar

- Research which is freely available for redistribution

Submit your manuscript at www.biomedcentral.com/submit 